G416(P) A HOSPITAL-BASED SURVEY OF ORAL HEALTH KNOWLEDGE AND PRACTICES OF PARENTS AND CARERS IN DERBYSHIRE

${ }^{1,2} \mathrm{Q}$ Ong, ${ }^{1} \mathrm{C}$ Utting, ${ }^{3} \mathrm{~S}$ Whiston, ${ }^{4} \mathrm{M}$ Braithwaite, ${ }^{1} \mathrm{H}$ Sanders, ${ }^{2} \mathrm{O}$ Okike, ${ }^{5,6} \mathrm{~A}$ Dickenson. ${ }^{1}$ Community Dentistry, DCHS NHS Foundation Trust, Derby, UK; ${ }^{2}$ Paediatrics, Derby Teaching Hospital NHS Trust, Derby, UK; ${ }^{3}$ Dental Public Health, Public Health England, Mansfield, UK; ${ }^{4}$ Oral Health Promotion Team, Derbyshire Community Health Services NHS Trust, Derby, UK; ${ }^{5}$ Maxillofacial Surgery, Derby Teaching Hospital NHS Trust, Derby, UK; ${ }^{6}$ Postgraduate Dental Dean, Health Education England, Leicester, UK

10.1136/archdischild-2018-rcpch.405

Background Tooth decay is largely a preventable disease. It affects almost a quarter of 5 -year-olds and was the most common reason for hospital admission for children aged 5-9 years in 2014-2015 in England. There is a huge financial burden of tooth decay on the NHS.

The aim of our survey was to ascertain the oral health knowledge and practices of parents and carers of children attending the Derbyshire Children's Hospital.

Method Parents or carers of children attending our hospital were surveyed between August and October 2017 using an anonymised paper-based questionnaire. It was developed using the 'Delivering Better Oral Health' document, which is an evidence-based toolkit published by Public Health England. The questions assessed knowledge, oral hygiene regime and dietary habits. Oral health information leaflet was provided after completion of the questionnaire.

Results Derbyshire Children's hospital is a busy District General hospital with $\sim 87000$ visits in 2016. A total of 800 surveys were completed representing about $1 \%$ of our expected attendance per year.

Most, 414 (52\%) were $\geq 7$ years old, whilst 217 (27\%) were 0-3 years and $166(21 \%)$ were 4-6 years old. Only 187/800 $(23 \%)$ of children were reported to be brushing their teeth correctly. Additionally, 114/383 (30\%) of children $\leq 6$ years of age do not regularly receive appropriate help with brushing. This was significantly worse amongst $4-6$ year olds, $p=0.006$. Although 711 (89\%) of parents/carers were aware of free NHS dental care for children, only 246 (31\%) visited or planned to visit a dentist at the earliest opportunity i.e. as soon as teeth erupt. Parents/carers reported that 172 (22\%) of children had unhealthy snacking habits.

Conclusion To our knowledge, this is the first hospital based survey in England. There appears to be gaps in the knowledge of parents/carers regarding good oral health. Our survey also highlighted unhealthy dietary habits with potential implications on oral health and wider impact on health. Oral health should remain in everyone's agenda to enable every child grow up free from tooth decay and have the best start in life. We propose oral health promotion and education for parents, carers and children. Hospital visits provide an excellent opportunity.

\section{G417(P) THE IMPACT OF NON-24 HOUR BLOOD CULTURE INCUBATION AND REPORTING ON THE NEONATAL UNIT}

A Anosike. Neonates, Royal Bolton Hospital, Bolton, UK

\subsection{6/archdischild-2018-rcpch.406}

Introduction A plan to centralise microbiology services in the region has led to a proposal to instal a local incubator near NICU or Paediatric ward. This due to the general consensus that 24 hour blood culture incubation and reporting will improve our clinical care and shorten the duration of antibiotic therapy and hospital stay of patients. Currently, laboratory staff will incubate blood culture samples only during working hours of 8-12 hours. Negative blood culture at 36 hours, with normal infection markers will allow us to stop antibiotic therapy where a baby is not septic.

Aim To assess the implication of non-24 hour blood culture incubation and reporting on clinical management of babies on the neonatal unit.

Method This was a retrospective audit carried out over 3 weeks. Information was sourced from Laboratory reports, prescription charts and medical notes. Babies admitted from other hospitals, whose blood cultures had already been taken were excluded.

Results 28 babies were audited from NICU, HDU and SCBU. 22 were screened for early onset sepsis, and 6 babies screened for late onset sepsis. 1 positive blood culture result found. 22 babies had normal CRP at the time of screening and 1224 hours after the start of antibiotics.

- Time of blood culture taken to start of incubation-

Range: 1 hour 16 min - 13 hours 10 mins

Average: 7 hours 13 mins

- Time of blood culture taken to 36 hours incubation-

Range: 36 hours 50 mins - 49 hours 18 mins

Average: 43 hours

- Duration of antibiotics therapy:

- 5 or more days for 7 babies

- 60 hours for 4 babies

- 36 hours for 2 babies

- 48 hours for 14 babies

Conclusion Non-24 hour incubation and reporting of blood cultures affect the duration of antibiotic therapy. It has no significant impact on the hospital stay on the neonatal unit. However, a similar audit on the postnatal and paediatric ward will show a significant effect of antibiotic duration on hospital stay and its cost to the trust. A local incubator will be essential to the neonatal and paediatric unit.

\section{British Academy of Childhood Disability and British Association for Child and Adolescent Public Health}

\section{G418 THE CLINICAL SPECTRUM OF DORSAL STREAM DYSFUNCTION IN AUTISM - A RETROSPECTIVE COHORT STUDY OF 13 CHILDREN}

${ }^{1} \mathrm{IJN}$ Hay, ${ }^{2} \mathrm{D}$ Assheton, ${ }^{3} \mathrm{~S}$ Biggar, ${ }^{4} \mathrm{H}$ Ibrahim, ${ }^{5} \mathrm{GN}$ Dutton. ${ }^{1}$ Community Paediatrics, NHS Dumfries and Galloway, Dumfries, UK; ${ }^{2}$ Paediatric Ophthalmology, St Helens and Knowsley Teaching Hospitals NHS Trust, Knowsley, UK; ${ }^{3}$ Educational Psychology, Dumfries and Galloway Council Education Services, Dumfries, UK; ${ }^{4}$ Ophthalmology, Mansoura University, Mansoura, Egypt; ${ }^{5}$ Visual Science, Glasgow Caledonian University, Glasgow, UK

10.1136/archdischild-2018-rcpch.407

Aims Bilateral dysfunction of the parieto-occipital cortex, linked to the visual cortex by the dorsal stream, variably produces simultanagnosia (SIM), optic ataxia (OA), and gaze apraxia. This triad, rarely reported in childhood, comprises 'Balint syndrome'. 'Dorsal stream dysfunction' (DSD) describes milder degrees of this disorder. 